\title{
Transformación y aprovechamiento de ayote Cucúrbita moschata como suplemento alimenticio para personas del corredor seco en Jalapa
}

\author{
Enma Yolanda Turcios Martínez \\ Ingeniera en alimentos \\ Ana Guisela Barrientos Godoy \\ Química Farmacéutica y Maestra en Docencia Universitaria \\ Emérita de Jesús Ortiz Lima \\ Licenciada en Trabajo Social y Maestra en Andragogía \\ Dirección General de Investigación -DIGI- \\ Centro Universitario de Sur Oriente -CUNSURORI- \\ Universidad de San Carlos de Guatemala
}

Fecha de recepción: 28/08/2014

Fecha de aceptación: 02/10/2014

\begin{abstract}
Resumen
En el departamento de Jalapa, los agricultores cultivan ayote Cucurbita moschata en asocio con frijol Phaseolus vulgaris y maíz Zea mays igual que en la época precolombina. Estos tres alimentos eran la base de la dieta de los mayas y los cultivaban, no sólo por el valor alimenticio sino también por la lógica ecológica que esto representa, ya que el maíz necesita mucho nitrógeno del suelo, el frijol lo fija de la atmósfera y el ayote recubre la tierra, evitando el crecimiento de malezas. En Jalapa se cultiva el ayote sólo con fines ecológicos sin darle importancia a las propiedades alimenticias, se desecha el $50 \%$ de los ayotes sazones que se cultivan, debido a su poca comercialización y utilización en la dieta. Se pretendió con esta investigación analizar organoléptica y nutricionalmente dos fórmulas de transformación del ayote, para luego estandarizarlas y dárselas a conocer a un área de la población afectada nutricionalmente en el corredor seco del departamento de Jalapa, y así puedan integrarlo a su dieta alimenticia o bien en el futuro comercializarlo para mejorar su nivel de vida y por ende su situación nutricional. Las dos formulaciones estandarizadas fueron harina y ensalada envasada para su prolongada conservación y posible comercialización. Los dos alimentos preparados fueron aceptados satisfactoriamente por la población en estudio con más del $80 \%$, y su valor nutricional fue significativo, sin cambios relevantes comparado con el valor inicial del ayote sin procesar. Concluyendo que ahora se cuenta con dos formulaciones para la transformación y aprovechamiento de Cucúrbita moschata como suplemento alimenticio para personas afectadas nutricionalmente en el corredor seco del departamento de Jalapa.
\end{abstract}

Palabras clave: Ayote, corredor seco, nutrición, suplemento alimenticio, dieta maya

\begin{abstract}
In the department of Jalapa, farmers grow a type of pumpkin called ayote Cucurbita moschata in association with bean Phaseolus vulgaris and corn Zea mays as in pre-Columbian times. These three crops were the staple diet of the Mayans and cultivated, not just the nutritional value but also the ecological logic that this represents. Corn needs a lot of nitrogen in the soil, beans fixes from the atmosphere and ayote overlying the earth, preventing the growth of weeds. In Jalapa the ayote is grown only for ecological purposes without giving importance to food properties, $50 \%$ of this kind of pumpkins that are grown every seasons is discarded, due to their poor marketing and use in the diet. This research was intended to analyze organoleptic and nutritionally two transformation formulas of ayote and then standardize them and make them accessible to an area of the population affected by nutritionally in Jalapa, so they can integrate it into their food or diet commercialize the future to improve their standard of living and therefore their nutritional status. The two formulations were standardized and packaged as salad meal for prolonged preservation and marketing. The two prepared foods were successfully accepted by the study population with over $80 \%$, and their nutritional value was significant, with no significant changes compared with the initial value of raw ayote. Concluding that now there are two formulations for processing and utilization of Cucurbita moschata as a food supplement for nutritionally affected in the dry corridor of the department of Jalapa.
\end{abstract}

Key words: Ayote, dry corridor, nutrition, nutritional supplement, mayan diet 


\section{Introducción}

El ayote Cucúrbita moschata es una planta anual y cultivada, que se maneja tanto en el sistema de agricultura tradicional de temporada, como en el de riego, presentando diversas variantes en tiempo para la aparición de flores y frutos. En Guatemala, de manera general, se siembra al inicio de la época de lluvias entre mayo y junio, floreciendo de julio a septiembre y fructificando de octubre a diciembre. Los frutos son de tamaño muy variable y formas diversas.

En el departamento de Jalapa se cultiva de forma similar a la que hacían los mayas en la época precolombina, sembrando maíz en asocio con frijol y ayote, en donde los tres cultivos en conjunto forman parte de la dieta alimenticia y a la vez se ayudan ecológicamente.

Esta forma de cultivo de los mayas se ha ido modificando y ahora ya no se siembra con fines alimenticios sino se le da prioridad al cultivo del maíz, seguido por el cultivo del frijol; pero el cultivo del ayote no ha tenido la misma importancia, ya que se utiliza con fines puramente ecológicos para favorecer el cultivo del maíz, y se ha ido dejando de lado su importancia como alimento con valor nutricional significativo, que en las buenas prácticas culinarias podría ayudar a contrarrestar la problemática de desnutrición que se ha venido dando en el corredor seco del departamento de Jalapa, ya que cuando termina la cosecha de maíz los agricultores se encuentran con una cantidad considerable de ayotes Cucúrbita moschata que poco a poco se van deteriorando hasta que los desechan. Según investigaciones realizadas en el país y fuera del país, el ayote Cucúrbita moschata es una gran fuente de potasio, vitamina $\mathrm{C}$, carbohidratos, grasa fibra y beta caroteno que es esencial para la visión, el buen estado de la piel, el cabello, las mucosas, los huesos y para el buen funcionamiento del sistema inmunológico, además de tener propiedades antioxidantes (Peter, 2006).
Por lo que se pretendió con esta investigación, transformar el ayote sazón Cucúrbita moschata en por lo menos dos formulaciones y estudiar su conservación y luego se analizó su valor nutritivo y aceptabilidad organoléptica, se socializó a la población afectada por el corredor seco para que el ayote sazón Cucúrbita moschata sea parte de su dieta alimentaria y ayudar así a contrarrestar la desnutrición y la aparición de enfermedades por falta de alimento, o bien que en el futuro lo puedan comercializar ya transformado y conservado correctamente.

Con los resultados de esta investigación sobre la transformación y aprovechamiento de ayote Cucúrbita moschata como suplemento alimenticio para personas afectadas nutricionalmente en el corredor seco del departamento de Jalapa, se lograron los objetivos que eran principalmente, encontrar por lo menos dos formulaciones para consumo humano de ayote, que no fueran las tradicionales utilizadas por la población guatemalteca, que conservaran el valor nutritivo natural del mismo, siendo ellas: harina que puede ser utilizada en sopa, atol, o como espesante, y ensalada envasada, luego de elaboradas y estandarizadas las formulaciones, se procedió a hacer el análisis sensorial, a través de un panel de catación, utilizando una boleta hedónica de caritas por tratarse de personas no lectoras en su mayoría, obteniendo resultados satisfactorios para la aceptación de color, olor sabor.

A la vez se hizo el análisis nutricional en laboratorios especializados, donde se obtuvieron resultados satisfactorios, ya que la mayoría de nutrientes se conservaron después del proceso de deshidratado y molido, así como el proceso de cocción leve para la ensalada. Por lo que ahora se cuenta con dos formulaciones para la utilización y aprovechamiento del ayote acumulado por los productores de maíz del área en el corredor seco del departamento de Jalapa. 


\section{Objetivos}

\section{- General}

Determinar la aceptabilidad del ayote sazón Cucúrbita moschata transformado, en alimentos con características organolépticas significativas, aprovechando su potencial nutricional, y ayudar así a contrarrestar la desnutrición de la población afectada en el corredor seco del departamento de Jalapa.

\section{- Específicos}

Estandarizar dos fórmulas de transfor mación y conservación del ayote sazón Cucúrbita moschata, que se consi- deren aceptables organoléptica y nutricionalmente, para el ser humano, a través de la experimentación.

Medir el grado de aceptabilidad de los dos alimentos preparados, utilizando el panel de catación.

Sensibilizar a la población afectada nutricionalmente en el corredor seco, sobre la importancia de incluir en su dieta alimenticia, el ayote sazón Cucúrbita moschata disponible.

Determinar la factibilidad de la comercialización del ayote transformado y conservado adecuadamente.

\section{Metodología}

Para la elaboración de dos subproductos de Ayote Cucúrbita moshata se revisó bibliografía acerca de estudios ya existentes sobre el valor nutritivo de esta cucurbitácea. Luego, se obtuvieron muestras para el análisis nutricional de ayotes que no tuvieran más de 6 meses almacenados.

Se realizó el análisis nutricional del ayote sazón Cucúrbita moschata, cultivado en el corredor seco del departamento de Jalapa y se compararon los resultados obtenidos con el valor nutricional encontrado en la revisión bibliográfica, en donde además se obtuvo información sobre la transformación y conservación de alimentos provenientes de hortalizas para que posteriormente se elaboraran 3 fórmulas artesanales en base a la información encontrada bibliográficamente, siendo estas harina, ensalada y snaks.

Se eligieron 3 fórmulas que se acoplaran a las características del ayote sazón Cucúrbita moschata, y que pudieran realizarse artesanalmente.

Además, se llevaron a la práctica experimental las fórmulas elegidas las veces que fueron necesarias hasta lograr resultados esperados.

De las tres fórmulas, se seleccionaron las que se comportaron mejor organoléptica y nutricionalmente en la práctica experimental, siendo la harina y la ensalada envasada.

Siguiendo las características de la investigación que se realizó, se utilizó el análisis sensorial con la prueba discriminatoria sugerida, de aceptación o rechazo, a través de una boleta hedónica de caritas, se procedió a la obtención de los resultados cuantitativos utilizando la siguiente ecuación, que es la que sugirió el método de análisis sensorial utilizado:

$\%$ de aceptación $=$ Total respuestas positivas $\times 100$ o rechazo Total Jueces

Donde el universo fue la cantidad de mujeres y hombres de 7 comunidades del departamento de Jalapa, comprendidos entre 18 y 50 años de edad. La muestra estuvo formada por 92 personas de las comunidades correspondientes.

Con este análisis cuantitativo se pudo establecer si fueron aceptados o rechazados los subproductos elaborados a partir de ayote, tratándose de harina y ensalada respectivamente. 
Se realizó el análisis sensorial de aceptación o rechazo a través de un test hedónico que corresponde a la prueba de aceptabilidad, con catadores representativos de las comunidades afectadas en el corredor seco del departamento de Jalapa. Este tipo de análisis sugiere una boleta representada por caritas, por tratarse de personas adultas del área rural, que no todas saben leer y escribir, indicando a que se refiere cada una: me gusta, ni me gusta ni me disgusta, no me gusta

\section{Resultados}

El resultado del análisis nutricional de los dos alimentos estudiados comparados con el valor nutricional del ayote maduro sin procesar es el siguiente:

\section{Gráfica de las vitaminas encontradas en ayote maduro, harina de ayote y ensalada de ayote}

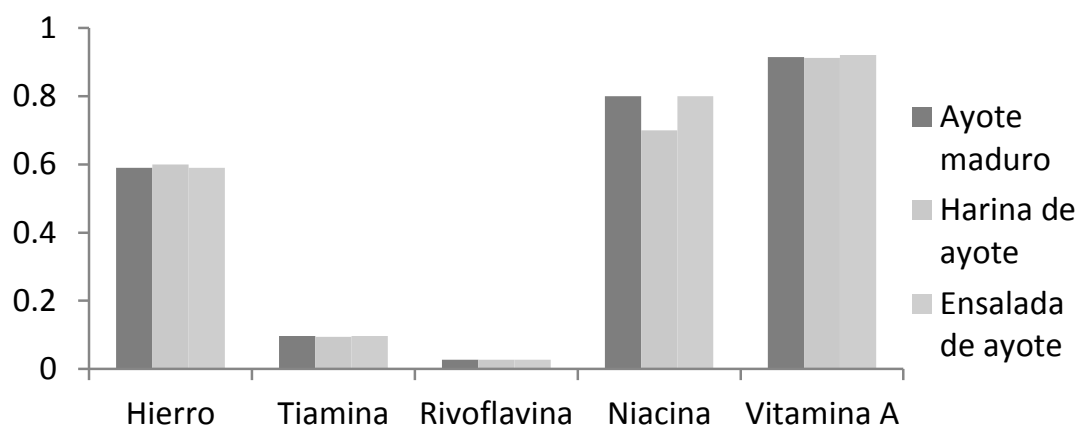

Gráfica 1

Se puede notar en la gráfica 1 que no hubo cambio significativo de los dos alimentos comparados con el valor nutricional del ayote sin procesar, una diferencia notable sin embargo, se observa en la Niacina para la harina, ya que se sabe que la Niacina puede ser destruida hasta un $30 \%$ expuesta al calor.

\section{Gráfica de minerales, grasas y vitamina $\mathrm{C}$ en ayote maduro, harina de ayote y ensalada de ayote}

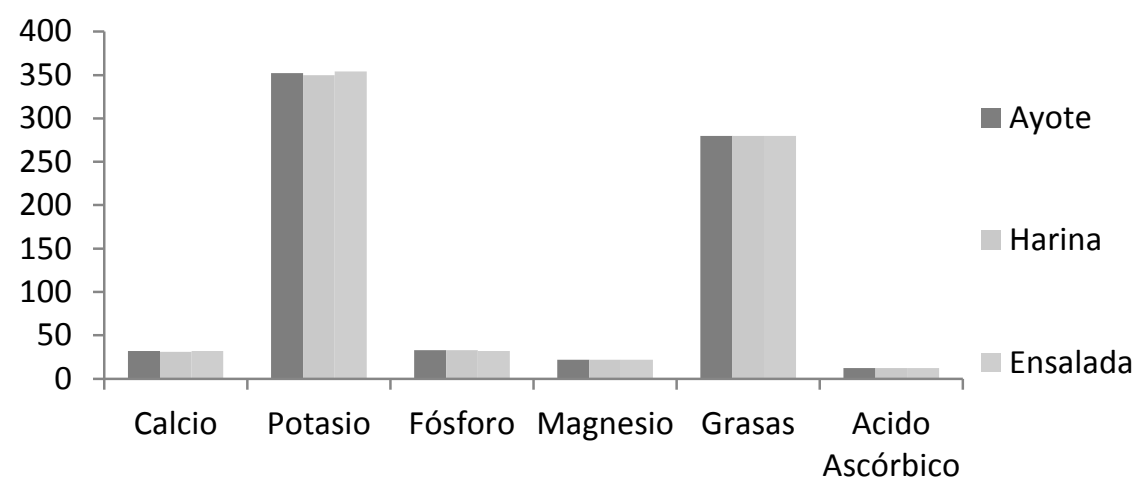

En la gráfica 2 se observa que no hubo diferencia significativa en los dos alimentos elaborados a partir de ayote maduro, comparados con el ayote maduro

\section{Gráfica 2}

sin procesar, por lo que se puede considerar el proceso como óptimo para esta clase de alimento. 


\section{Gráfica del valor nutricional de cenizas, proteínas, fibra cruda y carbohidratos}

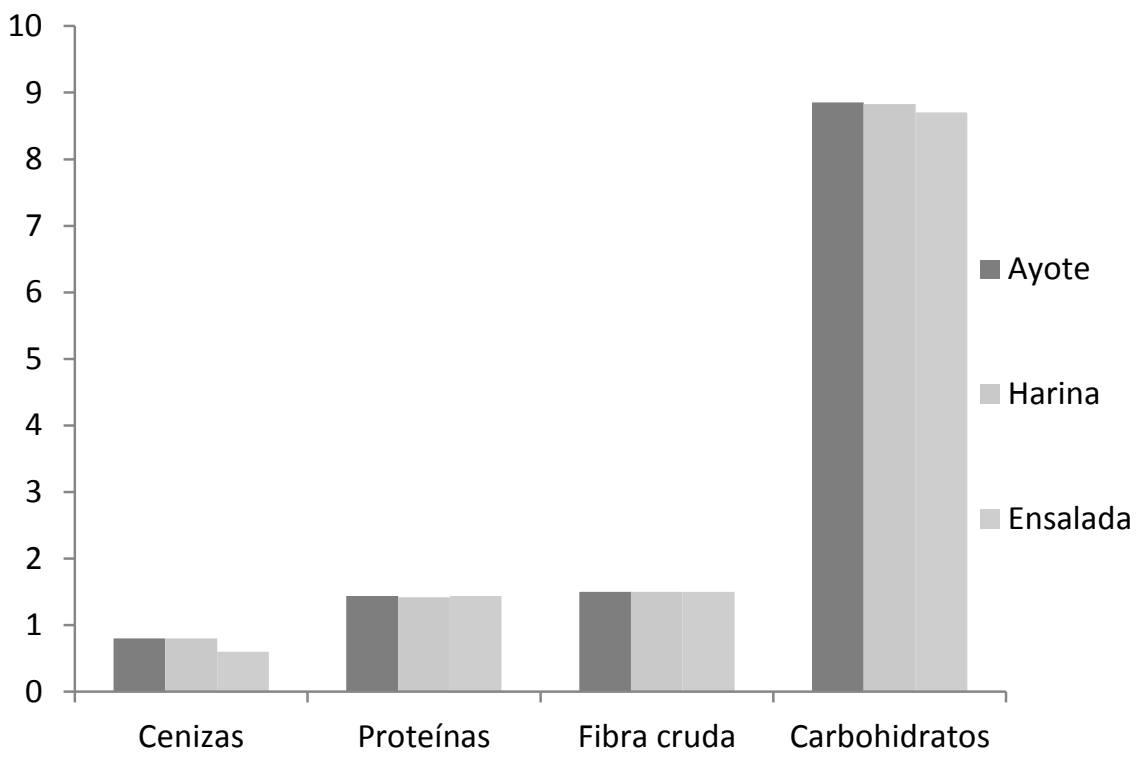

Gráfica 3

Se observa en la gráfica 3 que no hubo cambio significativo en el valor nutricional de cenizas, proteínas, fibra cruda y carbohidratos en harina de ayote y ensalada de ayote, respecto al valor nutricional del ayote sin procesar, considerando que el proceso utilizado fue el indicado para conservar el valor nutricional inicial de la materia prima.

\section{Aceptabilidad de la harina de ayote maduro}

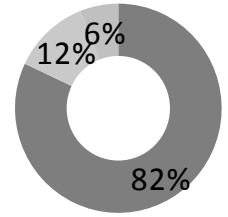

- Me gusta

Ni me gusta, ni me disgusta

\section{Gráfica 4}

La aceptabilidad de la harina de ayote maduro, fue en un $82 \%$. Se presentó a los catadores en forma de sopa, con mínima cantidad de sal. El $12 \%$ de los catadores dijeron que les era indiferente, que ni les gusta, ni les disgusta, y el $6 \%$ respondió que no es de su agrado, se puede ver en la gráfica que para la mayoría de los catadores fue aceptable en cuanto a sus condiciones organolépticas se refiere.

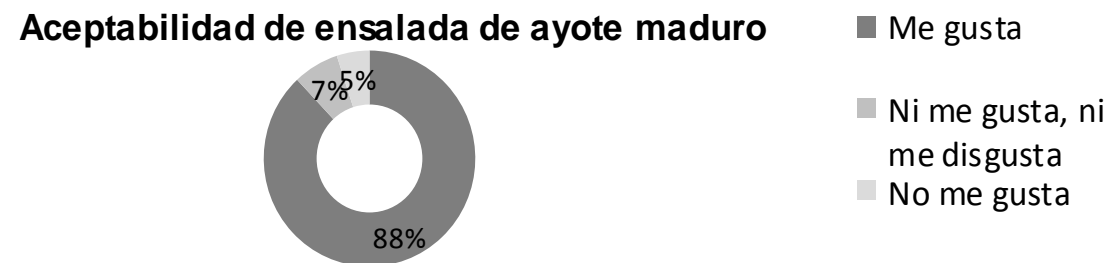

Gráfica 5 
Para la Ensalada de ayote la aceptabilidad fue del $88 \%$. El $7 \%$ indicó que ni le gusta, ni le disgusta, y el $5 \%$ opinó que no es de su agrado. Por lo que se puede ver que la ensalada de ayote fue aceptada por la

\section{Conclusiones}

Se lograron estandarizar dos fórmulas para transformar y conservar el ayote sazón, siendo la elaboración de harina, y elaboración de ensalada envasada para su conservación. Se logró elaborar cada una de las formulaciones sin la utilización de preservantes, conservando al máximo su valor nutricional.

El grado de aceptabilidad de los dos alimentos preparados fue del $82 \%$ para la harina de ayote, y del $88 \%$ para la ensalada, por lo que se puede concluir que dichos alimentos son agradables para los consumidores, por quienes fue aceptada satisfactoriamente.

Se sensibilizó a la población afectada nutricionalmente haciéndoles saber del valor alimenticio del ayote y lo accesible que es

\section{Recomendaciones}

Se recomienda darle seguimiento a la investigación en una segunda fase, para hacer el estudio de su factibilidad comercial, ya que la cantidad de ayote cosechado y almacenado en las viviendas productoras de maíz es en gran cantidad para consumirse en la alimentación de sólo una familia, y puede ser una alternativa para ayudar en el futuro a mejorar la economía familiar.

\section{Referencias}

- Bota, J (2001) Technology \& Engineering. mayoría de catadores participantes, ya que el sabor, color, olor son agradables a los sentidos, comparándolos con ensalada de otros vegetales con características similares.

para ellos aprovechar esta fuente de vitaminas, fibra, minerales, energía, etc. sin un costo elevado ya que se cultiva con fines ecológicos más no alimentarios. Además, se concluyó que al cocinar el ayote no se pierde el valor alimenticio como se puede observar en las gráficas correspondientes, a excepción de la niacina que se puede observar su disminución ligeramente, concluyendo que el calor solo afectó sobre la niacina en un porcentaje no significativo nutricionalmente.

Al haber obtenido los resultados de esta investigación, se puede concluir que se encontró una alternativa eficiente de transformar y aprovechar el ayote Cucúrbita moschata, conservando su valor nutritivo, proporcionando a la vez dos opciones distintas a las tradicionales de consumo humano.

- Ríus, et al. F. (2009). Bioéstadística: Métodos y Aplicaciones. Facultad de Medicina. Universidad de Málaga.

- Morales, R.G. (2007). Evaluación Sensorial aplicada a la investigación, desarrollo y control de la calidad en la Industria Alimentaria / Raúl G. Torricella Morales, Esperanza Zamora Utset y Horacio Pulido Alvarez. Ciudad de La Habana: Editorial Universitaria.

- Peter, F. (2006) Los alimentos: su elaboración y transformación. FAO.

- Zacca R. (2002). Morfología y fisiología de las plantas de zapallo, revista. 